\section{One Pythagoras for All Dimensions}

\section{Sasho Kalajdzievski}

$7 \mathrm{t}$ t is obvious that the Pythagorean theorem is equivalent to the following statement: the sum of the squares over the sides of a rectangle is equal to the sum of the squares over the diagonals (Fig. 1).

Diagonals in a box join opposite corners, as in Figure 2. It can be easily seen that the sum of squares over the edges of a box is equal to the sum of squares over the diagonals. In fact, the same statement holds true for all finite dimensions.

Theorem (Pythagorean theorem for all finite dimensions): The sum of the squares over the edges of any $n$-dimensional box is equal to the sum of the squares over the diagonals.

Proof. Any $n$-dimensional box $B^{n}$ can be viewed as the topological product $\left[0, a_{1}\right] \times\left[0, a_{2}\right] \times \cdots \times\left[0, a_{n}\right] \quad$ of intervals.

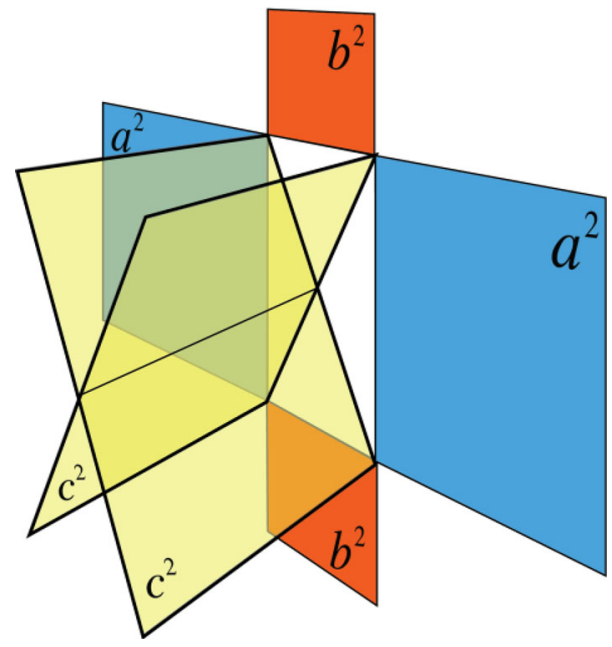

Figure I. Pythagorean theorem: $2 a^{2}+2 b^{2}=2 c^{2}$.

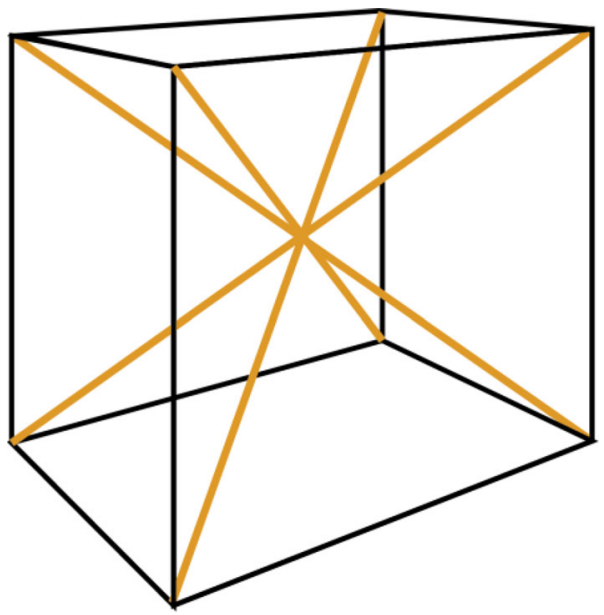

Figure 2. A 3-box and its 4 diagonals.

There are $n 2^{n-1}$ edges in $B^{n}$, joining corners $\left(x_{1}, x_{2}, \ldots, x_{i-1}, a_{i}, x_{i+1}, \ldots, x_{n}\right) \&\left(x_{1}, x_{2}, \ldots, x_{i-1}, 0, x_{i+1}, \ldots, x_{n}\right)$, $i=1,2, \ldots, n$, where each $x_{j}$ is in the set $\left\{0, a_{j}\right\}$. Out of these, there are $2^{n-1}$ edges of length $a_{i}, i=1,2, \ldots, n$. The sum of the squares over the edges is thus $2^{n-1}\left(a_{1}^{2}+a_{2}^{2}+\cdots+a_{n}^{2}\right)$.

Opposite corners in $B^{n}$ are points of type $\left(x_{1}, x_{2}, \ldots, x_{n}\right)$, $\left(y_{1}, y_{2}, \ldots, y_{n}\right)$, where $\left\{x_{i}, y_{i}\right\}=\left\{0, a_{i}\right\}$ for each $i=1,2, \ldots$, $n$. The length of each diagonal is $\sqrt{a_{1}^{2}+a_{2}^{2}+\cdots+a_{n}^{2}}$. There are $2^{n}$ corners, thus $2^{n-1}$ diagonals. So, the sum of the squares of diagonals is $2^{n-1}\left(\sqrt{a_{1}^{2}+a_{2}^{2}+\cdots+a_{n}^{2}}\right)^{2}$, as desired.

Department of Mathematics

University of Manitoba

Winnipeg, MB R3T 2N2

Canada

e-mail: sasho@cc.umanitoba.ca 